\title{
New electronic white cane for stair case detection and recognition using ultrasonic sensor
}

\author{
Sonda Ammar Bouhamed \\ Computer Imaging Electronic System (CEM Lab) \\ University of Sfax \\ Sfax Engineering School \\ BP W, 3038 Sfax, Tunisia
}

\author{
Imene Khanfir Kallel, Dorra Sellami Masmoudi \\ Computer Imaging Electronic System (CEM Lab) \\ University of Sfax \\ Sfax Engineering School \\ BP W, 3038 Sfax, Tunisia
}

\begin{abstract}
Blinds people need some aid to interact with their environment with more security. A new device is then proposed to enable them to see the world with their ears. Considering not only system requirements but also technology cost, we used, for the conception of our tool, ultrasonic sensors and one monocular camera to enable user being aware of the presence and nature of potential encountered obstacles. In this paper, we are involved in using only one ultrasonic sensor to detect stair-cases in electronic cane. In this context, no previous work has considered such a challenge. Aware that the performance of an object recognition system depends on both object representation and classification algorithms, we have used in our system, one representation of ultrasonic signal in frequency domain: spectrogram representation explaining how the spectral density of signal varies with time, spectrum representation showing the amplitudes as a function of the frequency, periodogram representation estimating the spectral density of signal. Several features, thus extracted from each representation, contribute in the classification process. Our system was evaluated on a set of ultrasonic signal where stair-cases occur with different shapes. Using a multiclass SVM approach, recognition rates of $82.4 \%$ has been achieved.
\end{abstract}

Keywords-Electronic white cane; ultrasonic signal processing; ground-stair classification ;temporal representation of ultrasonic signal; frequencial representation of ultrasonic signal

\section{INTRODUCTION}

Domestic space is a complex environment that contains various obstacles of different types at different locations: right, left, top and bottom. Even for none visually impaired, the congestion of such obstacles, sometimes poses problems, so what about those with visual impairment? People with visual disabilities are often dependent on external assistance which can be provided by humans, trained dogs, or special electronic devices as support systems for decision making. Existing devices are able to detect and recognize objects that emerge on the floor, but a real risk is also coming from objects that are decreasing from the floor, as holes or descending stairs. Accordingly, we are motivated in this paper to develop an automatic vision tool to overcome these limitations.

Using a traditional white cane is a universal solution, allowing a less risky journey for blind people. Such a tool is used to explore the environment by a frontal sweep, or contact with the ground to detect the presence of an obstacle. However, this cane does not allow sufficient exploration of objects that are at the top or which are getting too closer. To this end, the realization of an electronic cane automating the detection and recognition of fixed and mobile obstacles can offer more security and comfort to blind persons. This can be done through the integration of various specific sensors, which are designed to provide several types of information such as obstacles form, dimension, color and distance from the user. Some solutions are already exist on the market such as: Laser Cane [1], Teletact[2], UltraCanne [3], K Sonar cane [4], Smart Cane [5], Isonic [6], Guide cane [7], Palm Sonar [8], SmartWand [10], etc. These products help visual impairment people by collecting information through sensors and then, transmitting recommendations to them, through vibration or sound messages. A classification of these canes with respect to the type of sensors employed for obstacles detection is presented in [11].

The major disadvantages of these solutions are:

1) They only detect obstacle existence and distance without specifying indication about their nature which is important for the user to know.

2) They are unable or inaccurate in detecting some obstructions that are not protruding but present potential threat such as descending stairs, holes, etc.

3) The system communicates its recommendations, through intensity or frequency variations. Thus feedback information is often sent to the user through vibration or sound signals. So a training course is needed to keep the user informed about how to understand and react in real time to alerts that are transmitted regarding the existence of obstacles as well as their recognition. On the one hand, such training can be sometimes more expensive than the product itself. On the other hand, it is often difficult and complex for the users to assimilate it properly. Furthermore, in the case, where information is transmitted as an acute sound, that may happens several times especially when the obstacle is very close, it may be embarrassing for the blind person when they are in public.

Therefore, our interest is specifically focused, on the development of an electronic tool using two types of sensors which are ultrasonic sensors and monocular camera. Our choice of these sensors takes into account theirs area of operation and their performances. Our choice also depends on several other factors as: cost, type of scene, type of obstacle to be detected, detection range and desired precision of the measurements. The main idea consists in merging data 
provided by the two sensor types to allow more accurate information, to be transmitted to the user via a Bluetooth module as a voice message specifying the object nature, characteristics and the distance between the detected obstacles and the device.

In this paper, we explore ultrasonic sensor potentials in object detection and mainly stairs recognition. This type of sensors has significant potential in robotic applications. Indeed, it has been widely used in collision avoidance systems and in localization and navigation of mobile robots. In addition to robotic application, ultrasonic sensors are used in many other applications in different fields such as echography in medical field, nondestructive testing of materials ... Advantages that encourage us to use ultrasonic sensors is the ease to obtain distance information from immediate objects without intensive processing which can considerably lighten the application. They are also able to perform under low visibility conditions making it ideal for night as well as day use. Thus, ultrasound sensor seems to be a good solution for our system to detect and recognize several objects. However, object recognition under different viewing conditions is still a challenge for autonomous systems. So, the motivation of our work is to challenge by applying only one ultrasound sensor for obstacle recognition taking into account the weaknesses of this sensor type.

Many features can be extracted from the ultrasonic signal, providing different information and descriptions that are used to describe the detected object.

The remainder of this paper is organized as follows: In section 2, we present the contribution in the literature and the flowchart for the study. Section 3 show the proposed system architecture design that includes software and hardware components, working principal and wearability performance requirements of proposed electronic cane system. The ultrasonic signal processing for obstacle detection as well as well-known approaches used in the literature for object recognition are shown in section 4 . Then, section 5 present the related work of stair cases detection and recognition, before detailing proposed algorithm of ultrasonic signal preprocessing, feature extraction and SVM classification. The evaluation of our approach is discussed in section 6, and we finalize this paper with conclusions and perspectives.

\section{CONTRIBUTION IN THE LITERATURE}

After intensive study of blind needs in Tunisia through a large survey conducted with the Tunisian Blind Association (URA-Sfax) [45], it seems that the white cane presentation should not be replaced by other forms even when we look at improving it by making it intelligent with automatic obstacle detection tool and recognition options. In fact, the white cane is the clearest indicator to others, about blind person presence. In the literature, this idea was confirmed by some of researchers.

Indeed, in many studies related to the implementation of electronic cane, many authors considered to attach some components onto the white cane [4][6][10].

The design of our electronic white cane architecture, in this context, is as crucial task as the choice of the different hardware components. For example, it is necessary to satisfy the electrical conduction between sensors, microcontroller and batteries. It is also necessary to determine the deviation angle of each sensor to be able to detect obstacles placed in front of the user.

To design a prototype of our electronic white cane Fig. 1 summarizes the different steps of our survey. We review in the next section related technologies regarding the visually impaired. The stair case detection task is also examined.

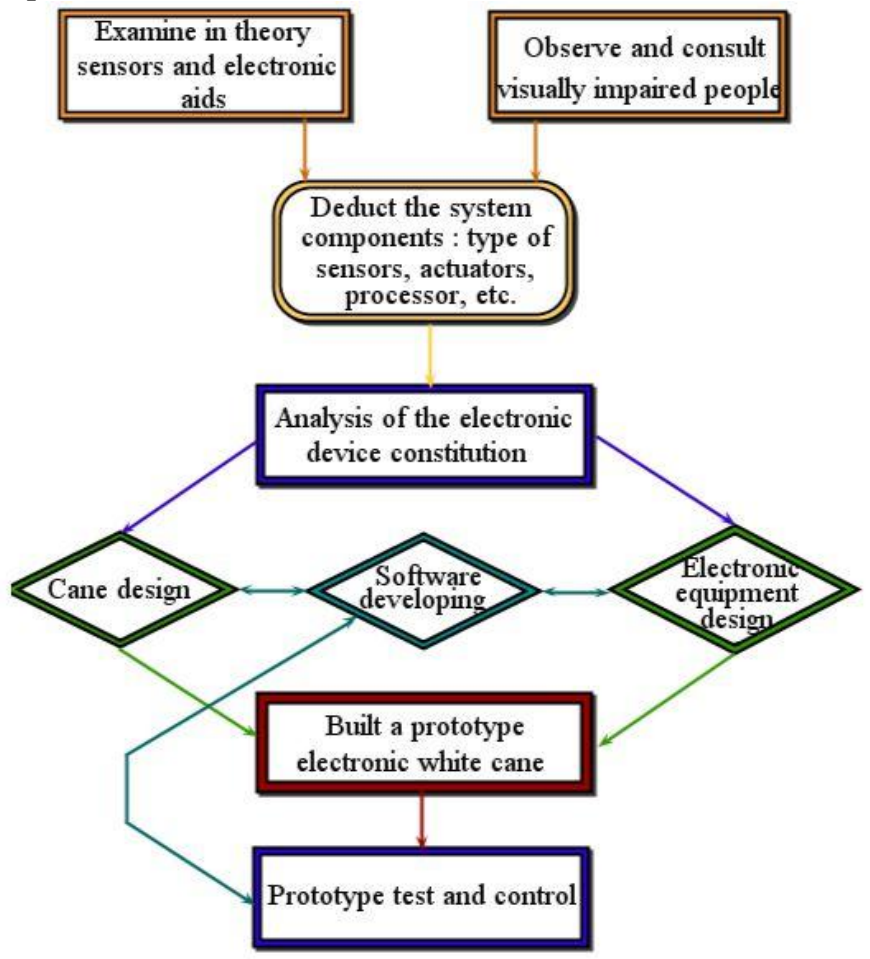

Fig. 1. Flowchart for the study

\section{SYSTEM DESIGN}

\section{A. Sensors}

\section{1) Different sensor types}

Sensors allow perception of the environment in more or less reliable way compared to the human eye. The use of different sensors is required, in different fields, to help the user in making a decision. Accordingly, we distinguish active and passive sensors.

A passive sensor measures a full energy provided by a physical phenomenon. In general terms, the sensors that use external energy sources to observe an object are called passive sensors. In the robotic world, the most used sensors are the Monocular cameras. They are inexpensive and efficient in terms of range, accuracy and amount of usable data.

Some systems use stereovision to detect and recognize objects. The principle is to infer information about the structure and distance in a 3D scene from two optical images taken from different viewpoints. It involves three stages: calibration, matching and triangulation. The mapping between the left and right images (registration) is the most crucial phase of processing.

An active sensor provides some kind of energy such as microwave, sound, light, etc., into the environment in order to 
detect the changes that occur on the transmitted energy. That means it transmits and detects at the same time. In the robotics world, this type of sensors is very robust for near and far obstacle detection. In addition, it determines an accurate measurement of the distance to the obstacle. The most used active sensors are ultrasonic, laser, and radar. Ultrasonic sensors work well for close obstacles unlike laser ones, which operate well for distant obstacles. Radar sensors are very robust for near and far obstacle detection, but their medium accuracy doesn't allow them detecting small obstacles. It is important to note that the sensor characteristics differ from one to another but make each sensor meeting specific requirements. Therefore, to achieve the best choice, we propose in the following, sensor characteristics comparison.

\section{2) Choice of the sensor}

The sensors selection must take into account the area of operation of each one and its performance. Also, it depends on several factors: detection range, cost, desired precision of the measurements, type of obstacle and type of scene.

Several constraints eliminate the use of stereo-vision in our system. Indeed, the stereo-vision processing is directly dependent on the accurate positioning and calibration of two cameras. Thus, at any variation, the error will automatically affects on the result.

Such variations are common in cane movement, and thus, results can be less accurate. Moreover, our application must meet the constraints of computing time. Indeed, aiming at facilitating the movement of blind people, the running time of our system has to be as short as possible in order to meet real time system requirements. Such requirement risks to be not supported by using stereo vision system as it should generate twice more images than monocular camera system. Therefore, the choice of a monocular camera makes sense since we want to implement a technique that aims to be:

- Fast (real time).

- Low cost.

- Precise and with acceptable range of vision.

Although, providing the richest information allowing recognition of detected obstacles, an optical sensor use, doesn't only raise the processing time a lot, but get also truncated information of the real scene. Indeed, data get from the camera doesn't provide distance information, being a detail of extreme importance for such an application. Therefore the use of a depth sensor is an ultimate necessity.

The choice of an active sensor depends on the measurement range of the sensor, , its response time, resolution, recognition reliability and finally the application requirements. For this end, a comparative survey is achieved and given in Table Iref $\{\mathrm{tab} 1\}$.

According to the survey results, shown in Table $\operatorname{Iref}\{$ tab1\}, the radar sensor is eliminated because it can neither detect small obstacles nor determine the distance to such objects. Thus, this sensor does not meet the requirements of our application.
TABLE I. GENERAL CHARACTERISTICS OF SOME ACTIVE SENSORS

\begin{tabular}{|c|c|c|c|}
\hline Laser & Radar & Ultrasound \\
\hline Principle & $\begin{array}{c}\text { Transmission } \\
\text { and reception of } \\
\text { light wave }\end{array}$ & $\begin{array}{c}\text { Transmission } \\
\text { and reception of } \\
\text { electromagnetic } \\
\text { wave }\end{array}$ & $\begin{array}{c}\text { Transmission } \\
\text { and reception of } \\
\text { ultrasonic waves }\end{array}$ \\
\hline Range & About 60 meters & About 250 m & $\begin{array}{c}\text { From 3 cm to 10 } \\
\text { meters }\end{array}$ \\
\hline Accuracy & High (about 5 & $\begin{array}{c}\text { Medium (few } \\
\text { meters) }\end{array}$ & $\begin{array}{c}\text { Very high }(5 \\
\text { mm) }\end{array}$ \\
\hline Price & Very high & high & Low \\
\hline
\end{tabular}

The proposed tool does not require a very large extent, that's why an increase from 3 to 4 meters is more than sufficient. In addition, our goal is to offer not only an efficient and reliable cane, but also a low cost one. In this case, the best sensor, which is closest to our needs, is the ultrasound one.

3) Sensor system model

The objectives that we project to meet in the present paper, assuming that the blind people are navigating in environment autonomously, are to:

- Generate the "Ascending or descending Stair case" through found signal.

- Define the distance, between the blind and the staircase, to be transmited to the user via a Bluetooth module as a voice message.

This work employed "LV-EZ0" ultrasonic sensor [25]. It can measure ranges from 0 inches to 254 inches (6,45-meters) and provides sonar range information from 6 inches up to 254 inches with 1 inch resolution. The interface outputs are pulse width output, analog voltage output, and serial digital output. We can choose one of the three sensor outputs. Ultrasonic sensors emit a high frequency pulse of $42 \mathrm{Khz}$. The packaging of the sensor is light and small enough $(19,9 \times 22,1 \times 16,4 \mathrm{~mm})$ to be fixed onto a cane without any inconvenience. The beam width of ultrasonic sensor is narrow enough so that the sensors do not interfere with each other while keeping their efficiency to detect any obstacles on the floor.

The used monocular camera is "LinkSprite JPEG Color Camera TTL Interface [12]. It has a small dimension $32 \mathrm{~mm} \mathrm{x}$ $32 \mathrm{~mm}$ to allow its integration into the cane without any inconvenience. It can capture ranges from 10 to 15 meters with a maximum viewing angle of 120 Degrees and produce JPEG images whose resolution is adjustable up to $640 * 480$. The monocular camera is powered from $3.3 \mathrm{~V}$ or $5 \mathrm{~V}$ and its power consumption varies between $80 \mathrm{~mA}$ and $100 \mathrm{~mA}$.

The camera position is defined such that it can detect obstacles from the top to the bottom of the field of vision. Accordingly, it is placed almost in the middle of the cane.

\section{B. Proposed system architecture}

With the above specified components, the proposed electronic white cane system will work in such principles: Ultrasonic sensors and monocular camera allow scene acquisition through different data nature. Signals provided by sensors are processed in a signal processing unit. Data collected 
from US sensor, is processed to provide depth information of the scene scanned according to a given direction. The resulting signal is then shared in as many segments as there are objects in the scene. For each segment we associate, not only, distance label, but also other specific labels, telling about some obstacle charcteristics, that we extract from ultrasonic signal, such as form, situation, material consistency, etc. Otherwise, images captured by the monocular camera, are also segmented and each of their region is labelled.

Some of labels got from each one of the two sensors are gathered to ensure registration of ultrasonic and optical data. With such a design, our system determines the distance from the obstacle as well as some of its characteristics using data from both sensors: camera and US sensor.

All the collected information about the scene are then analysed to make a decision that is returned to the user as a voice message revealing the nature of the obstacle and the distance towards it. This message is transmitted from the SD card to a headset using Bluetooth module. The proposed system overview is shown in Fig. 3.

The electronic white cane design configuration is shown in Figure Iref \{cane design\}. The cane is designed to be adjustable in height, to suit its user size. This height is considered as an input parameter of our system, as well as the angle \$beta $\$$ between the cane and the horizontal. But, in order to simplify a little the task of testing and validation, we set, for our prototype, the cane length at $90 \mathrm{~cm}$, and $\beta$ at $113^{\circ}$, by fixing the cane on a carriage according to this inclination. We install our ultrasonic sensor that is used to detect on ground obstacles, at angle $\alpha$ with the cane allowing detection of an obstacle, or more specifically of the beginning of a staircase, at the distance of $2 \mathrm{~m}$. Such a distance shall guarantee more security for the user.

The box containing the monocular camera is placed at distance $d 4$ from the cane handle. The distance between the cane and the eventual beginning of the staircase being set at 2 $\mathrm{m}$, we can define the orientation angle $\gamma$ of our camera to detect each obstacle on the floor.

The parameters used in the model are:

$\beta$ : The angle between the cane and the carriage,

$\mathrm{d} 2$ : The length of the cane,

d3 : The distance between the cane and the eventual beginning of the staircase,

d4 : The distance between the cane handle and the camera,

The following parameters are calculated using the parameters given above

d1 : The distance between the tip of ultrasonic sensor and the floor.

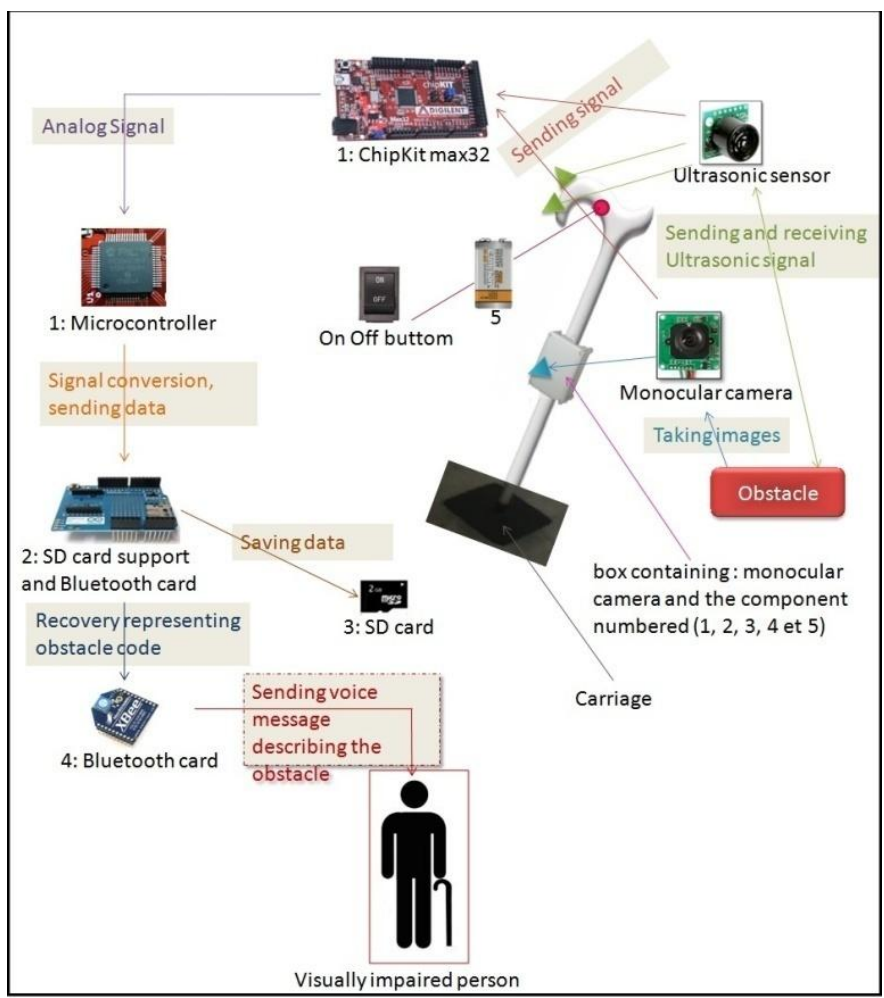

Fig. 2. Electronic cane system working principal

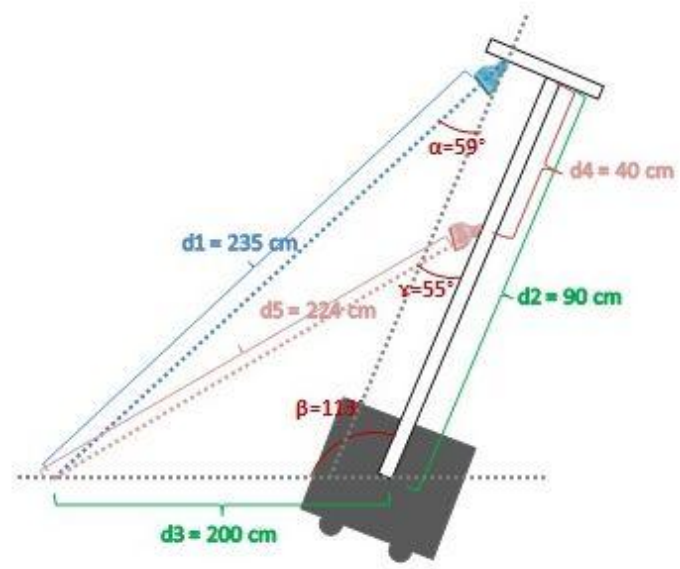

Fig. 3. Electronic white cane design configuration floor

d5 : The distance between the tip of the camera and the

$\alpha$ : The angle between the cane and the ultrasonic sensor

$\gamma$ : The angle between the cane and the camera.

To calculate the different sensor's angle inclination, we propose to use geometric rules within any triangle.

Let the triangle $A B C$ shown in Fig. 4. 


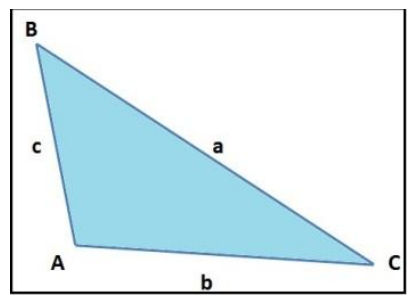

Fig. 4. Any triangle

To find the characteristics of the triangle, we use (1) to calculate the angles and (2) to determine the side lengths.

$$
\cos (A)=\frac{b^{2}+c^{2}-a^{2}}{2 . b . c}
$$

$$
a=b^{2}+c^{2}-2 \cdot b \cdot c \cdot \cos (A)
$$

we assume that the triangle has side dimensions $d l, d 2$ and $d 3$ and $\beta$ is the angle between sides of dimensions $d 2$ and $d 3$. So, to calculate $d l$ we use (2) and we obtain :

$\mathrm{d} 1=235 \mathrm{~cm}$

The same equation is used to calculate $d 5$ and we obtain : $\mathrm{d} 5=224 \mathrm{~cm}$

To calculate the angle $\gamma$, we assume that the triangle has side dimensions $d 3, d 5$ and $\mathrm{d} 6=\mathrm{d} 2-\mathrm{d} 4$. So, to calculate this angle, we use (1). We obtain $\gamma=0.55^{\circ}$.

The same equation is used to calculate the angle $\alpha$ and we obtain : $\alpha=0.59^{\circ}$.

\section{Wearability performance requirements}

The safety of visually impaired people imposes a reliable detection and recognition system. Many requirements have thus to be considered. Apart from electronic hardware and software concept, the wearability of the system is also a critical issue in our proposed system. The wearability requirements are:

\section{1) Usability}

The majority of electronic canes proposed in the literature requires training for their use. These courses are expensive and take long time. Aware of the importance of these details, the solution we propose saves on training costs due to its ease of use. It is our intention to provide essential information to the blind user with a simple tool that does not require any preliminary training.

\section{2) Robustness}

A System designed for people with visual impairments should be able to detect obstacles regardless variation of lighting conditions. Some risks may also occur during the use of the cane as his fall or it can be wet. To reduce the influence of these risks our cane should be anti-magnetic as well as being water resistant and shock resistant. Such a design has to be studied and managed.

\section{3) Efficiency and precision}

The system must reliably and precisely detect objects surrounding the blind regardless of their appearance, size and shape. Indeed, obstacles, missed in the detection step, expose the blind to a serious risk of accidents. Thus, our system is more effective as the number of errors, it might commit, is significantly reduced.

\section{4) Real-time system}

The term real time has several meanings depending on the context. In our context, we consider a system as 'real-time' one, if the information after its acquisition and processing remains relevant. The system must warn the visually impaired user so he can react in time.

\section{5) The cost}

The cost of the electronic white cane must be reasonable for all those requiring its use to help them in their day to day lives. The most popular electronic white canes proposed in the literature are very expensive since they use sophisticated sensors to have more efficiency. Unlike those products, our system relies on ingenious processing strategies, needing thus, only a single monocular camera, which is certainly less expensive than infrared camera or stereo vision systems as well as two ultrasonic sensors which the price is lower than the other active sensors such as radar and laser.

\section{6) Lightweight}

An embedded system on a white cane should not be cluttered with numerous sensors and large equipment which increases the weight of the cane. Several existing systems use many sensors which require a large box and increases the weight of the cane such as the Guide cane $\backslash$ cite $\{c 8\}$. The small weight of the traditional white cane allows users to easily scan their environment. Electronic white cane, is certainly heavier, but it should not prevent the scan so that the user can feel at ease as with his traditional cane.

It can be seen from the previous points, that the proposed solution is a device similar to the basic traditional white cane but with a set of sensors, interacting with each other to obtain an intelligent and efficient electronic white cane.

\section{Ultrasonic Signal Processing for Obstacle DETECTION}

\section{A. Ultrasonic signal}

Use ultrasonic signal processing is frequently used in nondestructive testing (NDT) of materials, medical characterization of tissues [26], construction industry [27], alimentary industry [28], in robotic application, etc. The classification steps depends strongly upon the features extracted to represent the object. Many properties can be extracted from ultrasonic registers. Although, there are some conditions where only trivial signal processing is required, there are some other cases where extracting these properties is a complex task.

Several information can be obtained from signal amplitude, but that doesn't necessarily provide the best representation of the signal [39]. Sometimes, the signal's frequency is more significant when more specific information are hidden in frequency components. Fourier Transform (FT) is often used for transforming the collected signal from time based signal to 
frequency-based one. The frequency-amplitude representation obtained by FT represents amplitude component for each frequency of the signal [30-40].

Numerous previous works have proposed various sets of ultrasonic features extracted from time and frequency domains and investigated the feasibility of using such parameters for an ultrasonic signal classification.

Time-domain ultrasonic features include principal components of signals [33] and estimates of the rectified signal envelope combined with various preprocessing methods: lowpass filtering, rectification, under-sampling, and meansubtraction [34].

In previous researches the most used feature in time domain are time of flight (TOF) information [31], echo energy [37], maximum amplitude of the echo [36], and correlation [29]. If we want to use only TOF information, for the classification, we need multi sensor system [38]. The echo amplitude is inadequate information, if it is used alone. The works presented by Dror et al. [32] established that the echo representation in the frequency domain gives the best results.

The various features, extracted from frequency-domain, which have been proposed by previous works [32], are statistical parameters extracted from statistical moments of an ultrasonic frequency spectrum such as, coefficient of skewness, coefficient of kurtosis [35], mean and coefficient of variance.

Combinations of time-domain and frequency- domain features also have been readily used [41-42].

After feature extraction steps, we need effective feature selection schemes to reduce the redundancy features and optimize their set.

This previous research has commonly traced the general guidelines of feature extraction from various domains of ultrasonic data analysis, that suggest the following steps:

- Extract as many descriptors (features) as possible from various domains.

- Evaluate their discrimination power with respect to the concerned classification problem.

- Choose the best set of features for classification.

\section{B. Object detection and recognition}

Our interest is exclusively focused on the use of ultrasonic sensors in our tool. To the best of our knowledge and from the latest research, ultrasonic sensors are not yet used to detect and recognize descending and ascending stairs.

We find in the literature, in the robotic field, some works concerning the classification of targets (corner, plane, cylinder and edge) using one ultrasonic sensor.

Firstly, [19] uses an artificial neural network to recognize two or three-dimensional shapes (cube and tetrahedron) independently from orientation, based on the echoes of ultrasonic pulses similar to those used by an echolocating bat.

Secondly, [20] presents the results of detection and classification of simply shaped objects using ultrasonic transducers. The subjects of object detection are an edge, a plan, a small cylinder and a corner using only one transducer and in indoor environment in mobile robotic applications.

Bozma and Kuc [22] introduced a concept for interpreting sonar TOF data obtained from specular surfaces. Physical properties of reflection and acoustic sensors are exploited to extract information about the environment in order to classify it in three ways (corner, plane and edge). This system uses a single mobile sensor for generating a sonar map.

Barat and Ait Oufroukh [21] developed statistical approaches for 2D target classification in an indoor environment using only the Time Of Flight (TOF), the maximum amplitude and 21 magnitudes to discriminate the different targets. This work classified targets in 4 ways(corner, plan, edge and small cylinder) using one transducer. II

In [23], Pham et al. provides a new application to monitoring activities of people in smart environments. Several scenarios were developed in which ultrasonic sensors were used for patient and elderly monitoring. Trajectory-matching algorithms were devised to classify people movement trajectories in indoor environments.

In [24], authors present an intelligent approach based on a 3D model of the environment, where the emphasis is on the extraction of features. In fact, researches have been primarily focused on determining walls and corners using ultrasonic sensors. Walls are considered as the extension of a line segment lying on a plane, whereas, corners are considered as the intersection of two planes, being observed from inside the concave space.

Most of works listed above, use more than one ultrasonic sensor to detect and recognize obstacles. Some others use single mobile ultrasonic sensor, that can obtain data from different points of view of the objects.

\section{Stair CASEs Detection AND ReCOGNition}

\section{A. Related Works}

Many approaches were described in the literature using different algorithms to detect and recognize wall, holes, descending and ascending stairs. Such systems are often essentially based on a laser sensor, infrared sensor or monocular camera.

Yuan and Manduchi [13] developed a hand-held environment discovery tool for the blind that integrates a laserbased range sensor. The user receives local range information when he swings the system around him. The time profile of the range is analyzed, by means of an extended Kalman filter, to detect environmental features that are critical to mobility, such as curbs, steps and drop-offs. This filter is used to track the range data and detect environmental features. In other work, Yuan and Manduchi [14] describe a new virtual white cane based on a laser pointer and a camera. These authors present in their paper a surface-tracking algorithm based on a JumpMarkov model for automatic detection of geometric singularities. This algorithm describes the evolution of range data in different types of surfaces, for example the foot of a wall, a step or a drop-off, by moving the system around and pointing it at different areas of the environment. 
Adams [15] introduced the new concept of an electronic cane for visually impaired people based on a combination of three infrared range sensors that were used to identify the terrain (even surface, ascending and descending stairs). The sensor system is close to the user's belt and it does not require swinging motion or any other movement by the user.

Lee and Lee [16] introduced the three infrared range sensor system for detecting, ascending and descending stairs. Decisions of the system are made based on current sensor readings. However, disturbance due to the user's movement was not considered.

Mihankhah [17] presents a theoretical analysis and implementation of autonomous staircase detection on a mobile robot. The robot is equipped with two laser sensors which scan the environment horizontally for the first and vertically for the second sensor.

Se and Brady [18] explain a distant stair case detection system, that uses optical camera or vision system camera to perceive outdoor environment.

Scherlen et al. [47] describe a new concept of Recognize Cane using a water detector, ambient humidity sensor and infrared sensors. This system can recognize the most common objects and environment clues like the soil humidity rate using a water detector and ambient humidity sensor. This system can also detect zebra crossings using brilliance sensor and a luminance sensor. The brilliance sensor is equipped with an infrared transmitter and receiver. This tool used two distant infrared sensors to recognize stairways or holes in the path of the user.

\section{B. Range of ultrasonic sensor}

The ultrasonic sensor provides four output formats which are pulse width output, analog voltage output, and serial digital output. The distance information $\$ \mathrm{~d} \$$ from the sensor tip to the obstacle can be obtained from the pulse width (PW) representation of range. Thereby, the distance value can be calculated using the scale factor of $147 \mathrm{uS}$ per inch. The sensor readings vary according to the terrain in our case, the floor or ascending or descending stair cases, as shown in Fig. 5.

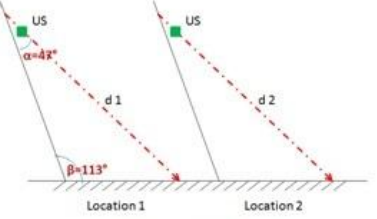

(a) Even surface

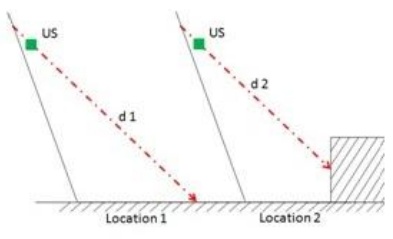

(c) Ascending stair 2

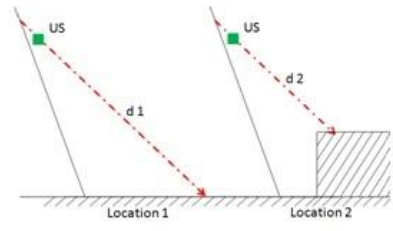

(b) Ascending stair 1

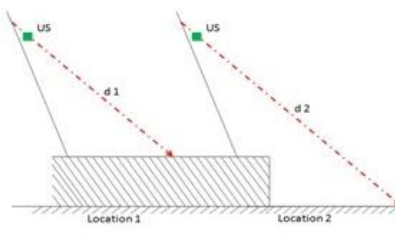

(d) descending stair 1
The three curves shown in Fig. 6 present the distance measures between the sensor and the nearest obstacle in three walking situations:

The top left curve shows the distance values when the user walks on a floor, without any change of floor state. The ultrasonic sensor outputs vary while the user walks, because the angle of incidence to the floor is large. So, it cannot provide accurate measurements.

The bottom curve shows the distance values when the user walks on a floor, then the cane detects an ascending stairs.

The top right curve shows the distance values when the user goes close to a descending stairs after an even surface.

Logically, the distance values must become larger (resp. smaller) than that obtained with a floor when the cane receives descending (resp. ascending) stairs. However, seeing curves of Figure $\mid r e f\{s o l\}$, it doesn't seem to be clear that the sensor readings change accordingly with floor states. Indeed, vibrations are common in cane movement resulting some errors in the ultrasonic output signal.

To separate the three cases experimental data identification rules of the floor state are developed in the following section.

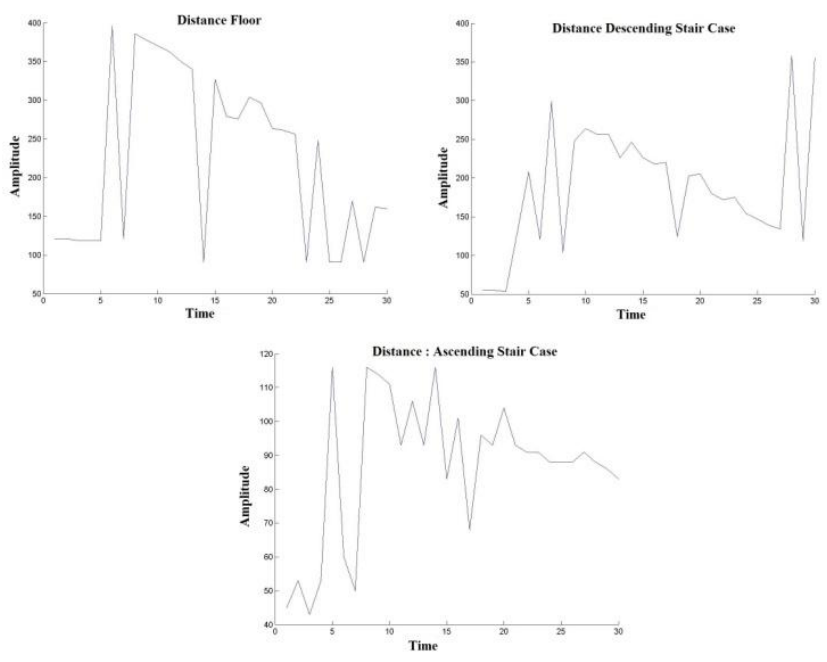

Fig. 6. Range sensor raw data - even surface (top left), ascending stairs (bottom) and descending stairs (top right)

\section{Preprocessing and Feature Extraction}

Since the ultrasonic sensor is attached to a cane, which is unstable due to the sweeping and tapping motions, enhancement of the ultrasonic sensor data is required. A low pass filter was used to filter ultrasonic registers. The use of the low pass filter has allowed eliminating the error in the ultrasonic signal.

Let us denote $x_{i}, i=0, \ldots N$, an ultrasonic signal. Several features was extracted from filtered signal in different domains.

- mean:

$$
\bar{x}=\frac{1}{N} \sum_{i=0}^{N} x_{i}
$$

Fig. 5. Sensor system and environment 
- Sample Standard Deviation:

$$
\sigma=\sqrt{\frac{1}{N-1} \sum_{i=0}^{N}\left(x_{i}-\bar{x}\right)^{2}}
$$

- Maximum.

- Minimum.

- The skewness (moment of order 3): is a measure of distribution symmetry around its mean.

$$
\mu_{3}=\frac{1}{N} \sum_{i=0}^{N}\left(x_{i}-\bar{x}\right)^{3}
$$

- The kurtosis (moment of order 4): is a measure of whether the data peakedness is relative to a distribution.

$$
\mu_{4}=\frac{1}{N} \sum_{i=0}^{N}\left(x_{i}-\bar{x}\right)^{4}
$$

- The root mean square (RMS): square root of the moment of order 2, being the variance that is given by (7). The RMS is a statistical measure of the varying quantity magnitude and it is given by (8).

$$
\begin{aligned}
& V=\frac{1}{N} \sum_{i=0}^{N}\left(x_{i}-\bar{x}\right)^{2} \\
& R=\sqrt{V}
\end{aligned}
$$

In the frequency domain, numerous features were calculated from different filtered signal representations as shown in Fig.9: the spectrum, the spectrogram and the periodogram.

The features extracted from the spectrum were the same features computed from the filtered signal in time domain. The spectrogram is a time-frequencial representation.

This time-frequency transform decomposes the signal $x$ over a family of time-frequency atoms $A_{t, f}$ where $t$ and $f$ are the time and the frequency localization indices. The resulting atom coefficients can be written as follows:

$$
F[t, f]=\sum_{i=0}^{N-1} x[i] A_{t, f}^{*}[i]
$$

where $*$ denotes the conjugate and the Short-time Fourier atoms $A_{t, f}$ shall be written as follows:

$$
A[i]=w[i-t u] \exp \left(\frac{i 2 \pi k i}{K}\right)
$$

where $w[i]$ is a Hanning window of support size $\mathrm{K}$.

The time-frequencial representation provides a good domain for signal representation and classification. In fact, this type of representation contains some details that cannot be seen in the temporal representation of ultrasonic signal.

The texture of the spectrogram representations contains distinctive patterns that capture different characteristics of the ultrasonic signals.

The time-frequencial representation provides an image that is used to extract Haralik's texture features [44] which are:

- Angular Second Moment:

$$
f_{1}=\sum_{i} \sum_{j} p(i, j)^{2}
$$

Where $i$ and $j$ are two different gray level. $p$ is obtained by calculating the number of times when a pixel with value $i$ is adjacent to a pixel with value $j$.

- Contrast:

$$
f_{2}=\sum_{n=0}^{N_{g}-1} n^{2}\left\{\sum_{i=1}^{N_{g}} \sum_{j=1}^{N_{g}} p(i, j)\right\},|i-j|=n
$$

Where $N_{g}$ is the gray level number in the 2D image.

- Correlation is given by:

$$
f_{3}=\frac{\sum_{i} \sum_{j}(i j) p(i, j)-\mu_{x} \mu_{y}}{\sigma_{x} \sigma_{y}}
$$

Where $\mu_{x}, \mu_{y}, \sigma_{x}$ and $\sigma_{y}$ are the means and std. deviations of, respectively, $p_{x}$ and $p_{y}$ being partial probability density functions.

- Sum of Squares or Variance:

$$
f_{4}=\sum_{i} \sum_{j}(i-\mu)^{2} p(i, j)
$$

- Inverse Difference Moment that is given by :

$$
f_{5}=\sum_{i} \sum_{j} \frac{1}{1+(i-j)^{2}} p(i, j)
$$

- Sum Average that is performed as follows:

$$
f_{6}=\sum_{i=2}^{2 N_{g}} i p_{x+y}(i)
$$

Where $x$ and $y$ are the input coordinates (row and column) in the co-occurence matrix, and $p_{x+y}(i)$ is the probability of cooccurence matrix coordinates summing to $x+y$.

- Sum Variance:

$$
f_{7}=\sum_{i=2}^{2 N_{g}}\left(i-f_{8}\right) p_{x+y}(i)
$$

- Sum Entropy: 


$$
f_{8}=-\sum_{i=2}^{2 N_{g}} p_{x+y}(i) \log \left\{p_{x+y}(i)\right\}
$$

- Entropy:

$$
f_{9}=-\sum_{i} \sum_{j} p(i, j) \log (p(i, j))
$$

- Difference Variance:

$$
f_{10}=-\sum_{i=0}^{N_{g}-1} i^{2} p_{x+y}(i)
$$

- Difference Entropy:

$$
f_{11}=-\sum_{i=0}^{N_{g}-1} p_{x-y}(i) \log \left\{p_{x-y}(i)\right\}
$$

- Information measure of correlation 1:

$$
f_{12}=\frac{H X Y-H X Y_{1}}{\max \{H X, H Y\}}
$$

- Information measure of correlation 2:

$$
f_{13}=\left(1-\exp \left[-2\left(H X Y_{2}-H X Y_{1}\right)\right]\right)^{1 / 2}
$$

Where

$$
X H Y=-\sum_{i} \sum_{j} p(i, j) \log (p(i, j))
$$

$H X, H Y$ are the entropies of $p_{x}$ and $p_{y}$

$$
X H Y_{1}=-\sum_{i} \sum_{j} p(i, j) \log \left\{\left(p_{x}(i) p_{y}(j)\right)\right\}
$$

$$
X H Y_{2}=-\sum_{i} \sum_{j} p_{x}(i) p_{y}(j) \log \left\{\left(p_{x}(i) p_{y}(j)\right)\right\}
$$

The features calculated from the filtered signal spectrum, were also extorted from the periodogram, in addition to other features that have been extracted from this representation:

- the variance performed according to (7);

- the biais (the moment of order 1):

$$
B=\frac{1}{N} \sum_{i=0}^{N}\left(x_{i}-\bar{x}\right)
$$

The whole frequency features constitute a 57 component feature vector.

\section{SVM Classification}

The performance of an obstacle categorization system depends on obstacle representation as well as on classification algorithm. In our system, we choose to apply SVM classifier in the classification task. SVM consists in a group of supervised learning methods that can be applied in classification. SVMs are used in many real-world applications such as text categorization, hand-written, character recognition, image classification, etc., and they are now established as one of the standard tools for machine learning and data mining [46]. The use of SVM classifier is interesting because it minimizes the bound taking into account empirical error and classifier complexity at the same time. In this way, SVMs are able of learning in sparse, high dimensional spaces with relatively few training examples [43]. They used an optimal hyper-plane as a decision function (Cf, Fig. 7). Thus, the optimal separating hyper-plane is used to classify an unlabeled input data, by using the following decision function:

$$
f(X)=\operatorname{sign}\left(\sum_{x_{i} \in S V}^{n}\left(y_{i} \alpha_{i} K\left(x_{i}, X\right)+b\right)\right)
$$

where $S V$ is the set of support vector items $x_{i}, b$ is the offset value, $K$ is the kernel, $\alpha_{i}$ are the optimized Lagrange parameters and $y_{i}$ is the label of $x_{i}, y_{i}$ may be 1 may be -1 .

The optimal separating hyper-plane is the one that maximizes the distance between itself and the nearest data point of each class as shown in Fig. 7.

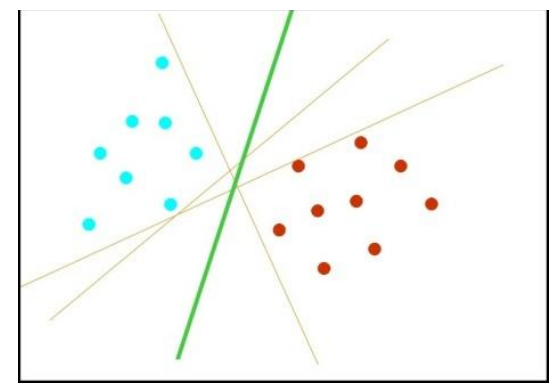

Fig. 7. The optimal separating hyper-plane

Different types of kernel can be used, RBF, Polynomial, etc... The kernel type affects the performance of SVM classifier.

In our system, we use RBF kernel which is defined as:

$$
K_{R B F}\left(x_{i}, x_{j}\right)=e^{-\left(\gamma\left\|x_{i}-x_{j}\right\|^{2}\right)}
$$

Where $x_{i}$ is the support vector, $x_{j}$ is the testing data point and $\gamma$ determines the area of influence this support vector has over the data space.

We consider three classes of indoor environment objects: even surface, descending stairs and ascending stairs.

For each category of features, an SVM classifier is trained to separate these classes by using one-against-one strategy.

\section{Evaluation OF THE Proposed ApPROACH}

Two raw data sets are constructed, the first is for the estimation of the optimal separating hyperplane parameters and the second for generalization, using the estimated optimal separating hyperplane. 
Fig. 10 and Fig. 11 show the signal preprocessing procedure, feature extraction and classification in, respectively, time domain and frequency domain.

Tab.II and Tab. III show the classification performances by use of ultrasonic signal in time domain for, respectively, the training data set and the generalization data set.

TABLE II. CLASSIFICATION RATES OBTAINED BY TRAINING DATA SET USING THE CONSIDERED TIME FEATURES

\begin{tabular}{|l|l|l|l|}
\hline \multicolumn{4}{|l|}{ (Output) Classified as: } \\
\hline $\begin{array}{l}\text { (Input) Known } \\
\text { as : }\end{array}$ & Even floor & $\begin{array}{l}\text { Ascending stair } \\
\text { cases }\end{array}$ & $\begin{array}{l}\text { Descending stair } \\
\text { cases }\end{array}$ \\
\hline Even floor & $0.46 \%$ & $0.43 \%$ & $0.11 \%$ \\
\hline $\begin{array}{l}\text { Ascending stair } \\
\text { cases }\end{array}$ & $0.05 \%$ & $0.95 \%$ & $0 \%$ \\
\hline $\begin{array}{l}\text { Descending stair } \\
\text { cases }\end{array}$ & $0.13 \%$ & $0.2 \%$ & $0.67 \%$ \\
\hline Accuracy & $70.73 \%$ & & \\
\hline
\end{tabular}

TABLE III. CLASSIFICATION RATES OBTAINED BY GENERALIZATION DATA SET USING THE CONSIDERED TIME FEATURES

\begin{tabular}{|l|l|l|l|}
\hline & \multicolumn{3}{|l|}{ (Output) Classified as: } \\
\hline (Input) Known as : & Even floor & $\begin{array}{l}\text { Ascending stair } \\
\text { cases }\end{array}$ & $\begin{array}{l}\text { Descending stair } \\
\text { cases }\end{array}$ \\
\hline Even floor & $0.45 \%$ & $0.40 \%$ & $0.15 \%$ \\
\hline $\begin{array}{l}\text { Ascending stair } \\
\text { cases }\end{array}$ & $0.09 \%$ & $0.91 \%$ & $0 \%$ \\
\hline $\begin{array}{l}\text { Descending stair } \\
\text { cases }\end{array}$ & $0.08 \%$ & $0.5 \%$ & $0.42 \%$ \\
\hline Accuracy & $60.26 \%$ & & \\
\hline
\end{tabular}

The results show a high ambiguity between even floor and ascending stair cases in the time domain. This problem is illustrated in Fig. 8 and Fig. 9 which represent the separating power of, respectively, "mean" and "maximum" features. We can clearly note the problem of no distinction between this two classes.

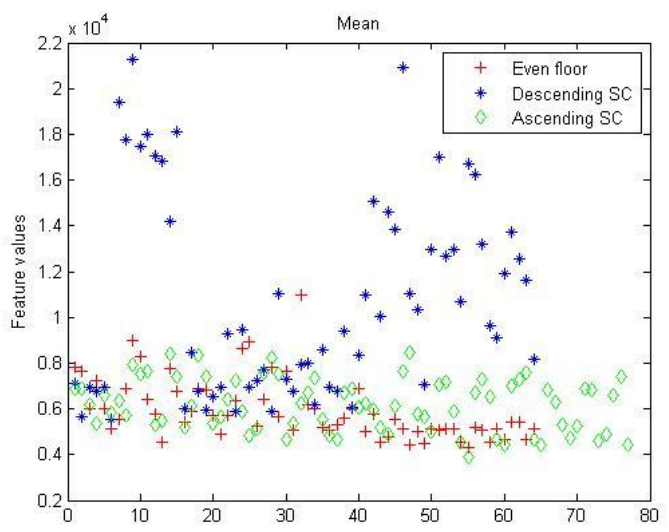

Fig. 8. The separating power of the 'mean' feature

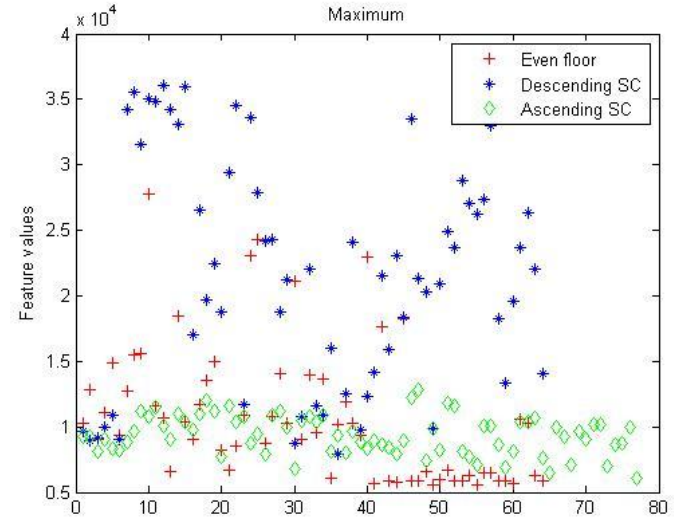

Fig. 9. The separating power of the 'maximum' feature

Such confusion is resolved by using the frequency domain features.

Tab.IV and Tab.V show classification performances of ultrasonic signal in frequency domain for the training data set and respectively for the generalization data set.

TABLE IV. CLASSIFICATION RATES OBTAINED BY TRAINING DATA SET USING THE CONSIDERED FREQUENCY FEATURES

\begin{tabular}{|l|l|l|l|}
\hline & \multicolumn{2}{|l|}{ (Output) Classified as: } \\
\hline $\begin{array}{l}\text { (Input) Known } \\
\text { as : }\end{array}$ & Even floor & $\begin{array}{l}\text { Ascending stair } \\
\text { cases }\end{array}$ & $\begin{array}{l}\text { Descending stair } \\
\text { cases }\end{array}$ \\
\hline Even floor & $0.75 \%$ & $0.19 \%$ & $0.06 \%$ \\
\hline $\begin{array}{l}\text { Ascending stair } \\
\text { cases }\end{array}$ & $0.10 \%$ & $0.90 \%$ & $0 \%$ \\
\hline $\begin{array}{l}\text { Descending stair } \\
\text { cases }\end{array}$ & $0.19 \%$ & $0.04 \%$ & $0.77 \%$ \\
\hline Accuracy & $80.97 \%$ & \multicolumn{2}{l}{} \\
\hline
\end{tabular}

TABLE V. CLASSIFICATION RATES OBTAINED BY GENERALIZATION DATA SET USING THE CONSIDERED FREQUENCY FEATURES

\begin{tabular}{|l|l|l|l|}
\hline & \multicolumn{2}{|l|}{ (Output) Classified as: } \\
\hline $\begin{array}{l}\text { (Input) Known } \\
\text { as : }\end{array}$ & Even floor & $\begin{array}{l}\text { Ascending stair } \\
\text { cases }\end{array}$ & $\begin{array}{l}\text { Descending stair } \\
\text { cases }\end{array}$ \\
\hline Even floor & $0.89 \%$ & $0.11 \%$ & $0 \%$ \\
\hline $\begin{array}{l}\text { Ascending stair } \\
\text { cases }\end{array}$ & $0.26 \%$ & $0.71 \%$ & $0.03 \%$ \\
\hline $\begin{array}{l}\text { Descending stair } \\
\text { cases }\end{array}$ & $0.32 \%$ & $0.12 \%$ & $0.56 \%$ \\
\hline Accuracy & $80.97 \%$ & & \\
\hline
\end{tabular}

The results obtained from classification in frequency domain show that the confusion between even floor and ascending stair cases are really weakened and it is clearly seen from these results that the best classification is obtained while projecting raw data on differents representation of ultrasonic in signal frequency domain. 
Each representation allows having information that cannot be obtained from the others representations. The fusion of this information reduces the imperfection of the data and improves the system's performance.

\section{Coarse to fine strategy}

In our system, we look at meeting not only efficiency and precision, but it is also necessary to ensure optimal processing time. Hence, we are motivated to reduce the running time of the system processing.

When a blind people navigates in his environment, he needs to be alerted only when the cane detects ascending or descending stair cases. So, it is not necessary to classify the environment on three classes every time. Indeed, we propose a strategy which is based on two levels of classification in order to speed up the classification process, without compromising recognition performance. The first level is ensured by a strong SVM classifier that classifies the environment on two classes Even floor and Not Even Floor. Meanwhile, the second level is optional as it is only used if the decision of the first level's classifier is "Not Even Floor" (Cf. Fig. 12 ).

Tab.VI and Tab.VII show the classification performances by use of ultrasonic signal in frequency domain, on, respectively, the training data set and the generalization data set, while based on the new approach.

Our second strategy allows not only to decrease the time processing but also it provides significant improvement of classification performances. We can thus deduce that solutions based on multiple classifiers are more general than those based on one classifier.

TABLE VI. CLASSIFICATION RATES OBTAINED BY TRAINING DATA SET WITH TWO LEVELS OF CLASSIFICATION

\begin{tabular}{|l|l|l|}
\hline & \multicolumn{2}{|l|}{ (Output) Classified as: } \\
\hline (Input) Known as : & Even Floor & Not Even Floor \\
\hline Even Floor & $0.63 \%$ & $0.37 \%$ \\
\hline Not Even Floor & $0.10 \%$ & $0.90 \%$ \\
\hline Accuracy & $80.97 \%$ & \\
\hline
\end{tabular}

TABLE VII. CLASSIFICATION RATES OBTAINED BY GENERALIZATION DATA SET WITH TWO LEVELS OF CLASSIFICATION

\begin{tabular}{|l|l|l|}
\hline & \multicolumn{2}{|l|}{ (Output) Classified as: } \\
\hline (Input) Known as : & Even Floor & Not Even Floor \\
\hline Even Floor & $0.85 \%$ & $0.15 \%$ \\
\hline Not Even Floor & $0.18 \%$ & $0.82 \%$ \\
\hline Accuracy & $82.76 \%$ & \\
\hline
\end{tabular}

Tab.VIII and Tab.IX show the classification performances, in ascending and descending stair cases, by use of ultrasonic signal in frequency domain, on, respectively, the training data set and the generalization data set, while basing on the new approach.

TABLE VIII. CLASSIFICATION RATES OBTAINED BY TRAINING DATA SET WITH TWO LEVELS OF CLASSIFICATION

\begin{tabular}{|l|l|l|}
\hline & \multicolumn{2}{|l|}{ (Output) Classified as: } \\
\hline (Input) Known as : & $\begin{array}{l}\text { Ascending stair } \\
\text { cases }\end{array}$ & $\begin{array}{l}\text { Descending stair } \\
\text { cases }\end{array}$ \\
\hline Ascending stair cases & $0.99 \%$ & $0.01 \%$ \\
\hline Descending stair cases & $0.06 \%$ & $0.94 \%$ \\
\hline Accuracy & $96.45 \%$ & \\
\hline
\end{tabular}

TABLE IX. CLASSIFICATION RATES OBTAINED BY GENERALIZATION DATA SET WITH TWO LEVELS OF CLASSIFICATION

\begin{tabular}{|l|l|l|}
\hline & \multicolumn{2}{|l|}{ (Output) Classified as: } \\
\hline (Input) Known as : & $\begin{array}{l}\text { Ascending stair } \\
\text { cases }\end{array}$ & $\begin{array}{l}\text { Descending stair } \\
\text { cases }\end{array}$ \\
\hline Ascending stair cases & $0.94 \%$ & $0.06 \%$ \\
\hline Descending stair cases & $0.16 \%$ & $0.84 \%$ \\
\hline Accuracy & $89.83 \%$ & \\
\hline
\end{tabular}

\section{CONCLUSIONS AND PERSPECTIVES}

Blinds and visually impaired people need some aid to interact with their environment with more security. Accordingly, a multi-sensor system that scans floor surfaces and detects the presence of stairs was developed.

In this paper, we have presented a new electronic tool that incorporates two ultrasonic sensors and one monocular camera, intended for visually impaired assisting. Only one ultrasonic sensor was used to detect and identify three floor states, even floor, ascending stair case and descending stair case. To this end, we developed an approach for detection as well as identification of floor states. Such performances are challenging, since no existing solutions has proposed detecting stairs. Besides, most of existing tools aiming to detect objects basing on ultrasonic measurements make use of a series of ultrasonic sensors. The recognition result is estimated to $82.7 \%$ for detecting stair presence and $89.8 \%$ for precising if it consists in either ascending or descending type.

The results of this study allowed us to prove how much using one ultrasonic sensor to recognize the floor state is interesting. The recognition result is not perfect, as it doesn't reach the zero error performance, that is crucial for the tool that we are developing, but it is sufficiently satisfactory to contribute in the decision.

Our future works will focus on this topic. Indeed, we are working on merging data captured from two different sources of knowledge, precisely ultrasonic sensor and monocular camera, to improve the system's performances. 


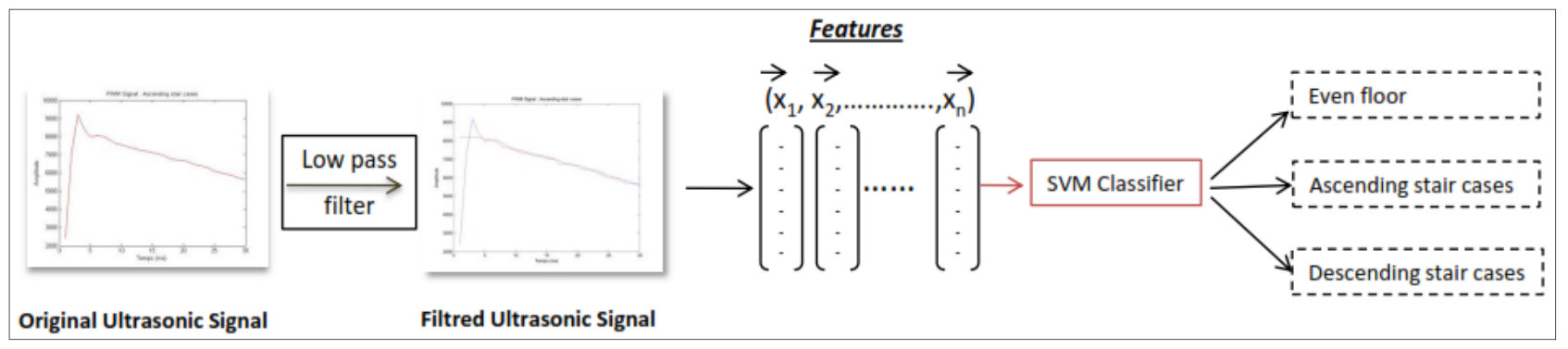

Fig. 10. Proposed strategy : Feature extraction and classification in time domain

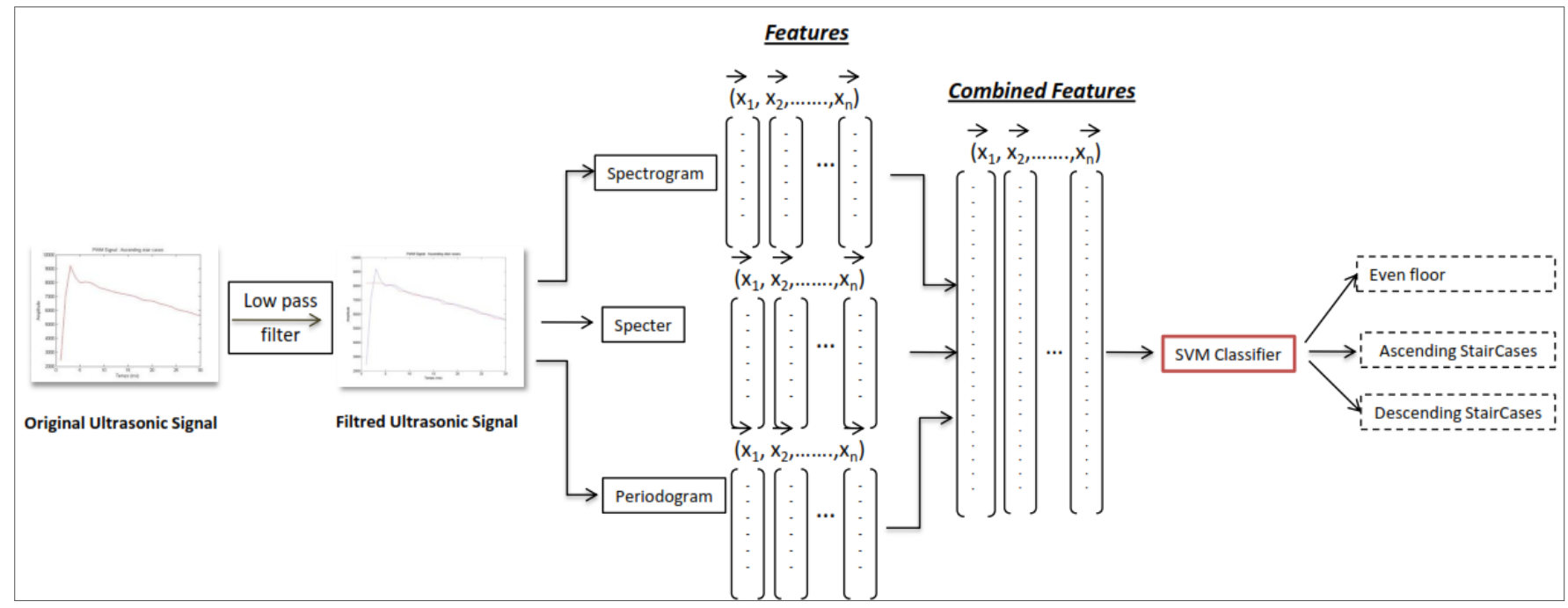

Fig. 11. Proposed strategy : Feature extraction and classification in frequency domain

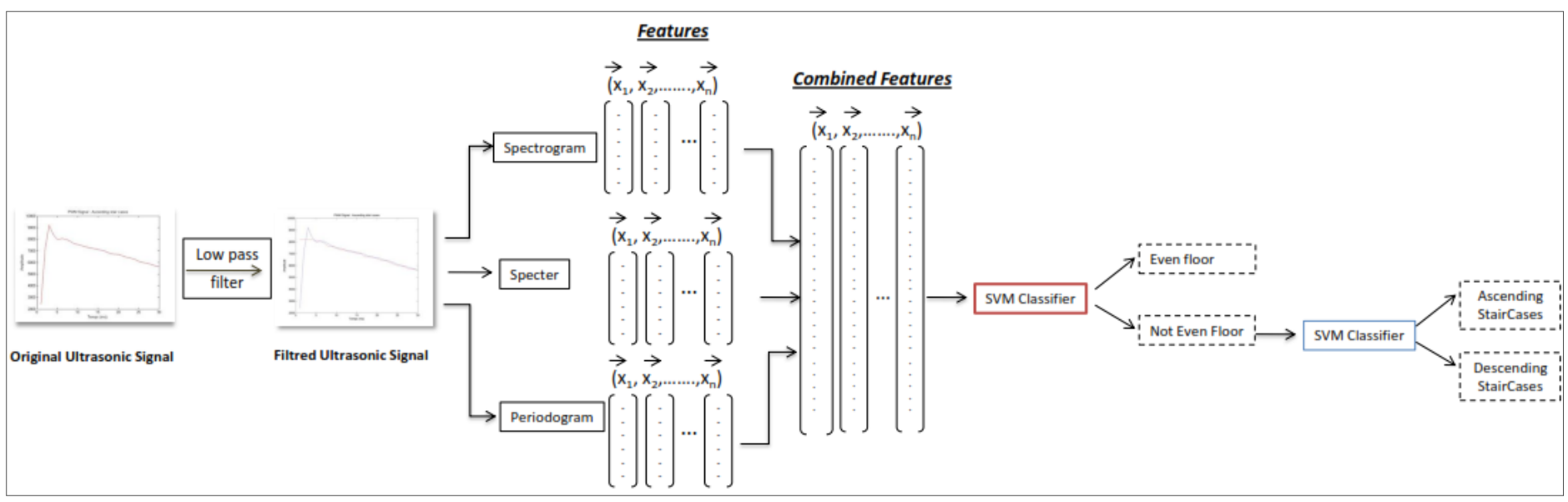

Fig. 12. Proposed strategy : two levels of classification in frequency domain 


\section{REFERENCES}

[1] J. M. Benjamin, N. A. Ali, and A. F. Schepis, "A Laser Cane for the Blind" Proceedings of the San Diego Biomedical Symposium, vol. 12, pp. 53--57, 1973.

[2] R. Farcy, R. Leroux, R. Damaschini, R. Legras, Y. Bellik, C. Jacquet, J. Greene and P. Pardo, "Laser Telemetry to improve the mobility of blind people: report of the 6 month training course", ICOST 2003 1st International Conference On Smart homes and health Telematics Independent living for persons with disabilities and elderly people, Paris, pp. 24--26, September 2003.

[3] B. Hoyle, D. Withington and D. Waters, "UltraCane", Available from: "http://www.soundforesight.co.uk/index.html", June 2006

[4] T. Terlau and W. M. Penrod, "K'Sonar Curriculum Handbook", Available from: "http://www.aph.org/manuals/ksonar.pdf", June 2008

[5] L. Whitney, "Smart cane to help blind navigate", Available from: "http://news.cnet.com/8301-17938\_105-10302499-1.html", 2009.

[6] E. Kee, "iSONIC cane for the virtually impaired", Available from: "http://www.ubergizmo.com/2011/01/isonic-cane-for-the-virtuallyimpaired/", 2011.

[7] J. Borenstein and I. Ulrich. "The GuideCane A Computerized Travel Aid for the Active Guidance of Blind Pedestrians". Proceedings of the IEEE International Conference on Robotics and Automation, Albuquerque, NM, pp. 1283--1288, Apr. 21-27, 1997.

[8] K. Takeuchi, "The Palm Sonar", Available from: "http://www.palmsonar.com/", 2010.

[9] MaxBotix, "LV-MaxSonar-EZ4 Data Sheet", Available from: "http://www.maxbotix.com/documents/MB1040\_Datasheet.pdf", 2005.

[10] S. Park, L. Kim, S. Ha, H. Cho and S. Y. Lee, "An Electronic Aid for a Visually Impaired Person Using an Ultrasonic Sensor", International Conference on Coastal Engineering (ICCE 2012), Las Vegas, pp. 10-14, Jan. 2009.

[11] S. A. Bouhamed, J. F. Elleuch, I. K. Kallel, D. S. Masmoudi, "New electronic cane for visually impaired people for obstacle detection and recognition", IEEE International Conference on Vehicular Electronics and Safety (ICVES), Istanbul, pp. 416--420, 2012.

[12] linksprite, DataSheet: "LinkSprite JPEG Color Camera Serial UART Interface", 2005.

[13] D. Yuan and R. Manduchi, "A Tool for Range Sensing and Environment Discovery for the Blind", Conference on Computer Vision and Pattern Recognition Workshop (CVPRW'04), 2004.

[14] D. Yuan and R. Manduchi, "Dynamic Environment Exploration Using a Virtual White Cane", IEEE Computer Society Conference on Computer Vision and Pattern Recognition (CVPR'05), Vol. 1, pp. 243--249, 2005.

[15] M. D. Adams, "On-Line Gradient Based Surface Discontinuity Detection for Outdoor Scanning Range Sensors", IEEE/RSJ International Conference on Intelligent Robots and Systems, pp. 1726-1731, 2001.

[16] M. Lee and S. Lee, "Design and analysis of an infrared range sensor system for floor-state estimation", Journal of Mechanical Science and Technology, pp. 1043--1050, 2011

[17] E. Mihankhah, A. Kalantari, E. Aboosaeedan, H. D. Taghirad, S. Ali and A. Moosavian, "Autonomous Staircase Detection and Stair Climbing for a Tracked Mobile Robot using Fuzzy Controller", Proceedings of the 2008 IEEE International Conference on Robotics and Biomimetics, Bangkok, Thailand, pp. 1980--1985, 2009

[18] S. Se and M. Brady, "Vision-based Detection of Stair-cases", Fourth Asian conference on computer Vision, ACCV 2000, Vol. I, pp. 535-$540,2000$.

[19] I. E. Dror, M. Zagaeski and C. F. Moss, "Three-dimensional target recognition via sonar: a neural network model", Neural Networks, Vol. 8, No. 1, pp. 149--160, 1995.

[20] J. M. Oria and A. M. G. Gonzalez, "Object recognition using ultrasonic sensors in robotic application", IECON, 19th Annual Conference of IEEE Industrial Electronics, pp. 1927--1931, 1993.

[21] C. Barat and N. Ait Oufroukh, "Classification of indoor environment using only one ultrasonic sensor", IEEE Instrumentation and Measurement Technology Conference, 2001.

[22] O. Bozma and R. Kuc, "Building a sonar map in a specular environment using a single Mobile sensor", IEEE TRANSACTIONS ON PATTERN ANALYSIS AND MACHINE INTELLIGENCE. Vol. 13, NO. 12, pp. 1260--1269, 1991.
[23] V. T. Pham, Q. Qiu, A. A. P. Wai and J. Biswas, "Application of ultrasonic sensors in a smart environment", Pervasive and Mobile Computing, Vol. 3, pp. 180--207, March 2007.

[24] G. N. Marichal, A. Hernández, L. Acosta and E. J. González, " A NeuroFuzzy System for Extracting Environment Features Based on Ultrasonic Sensors", Sensors, Vol. 9, pp. 10023--10043, 2009.

[25] MaxBotix, "LV-MaxSonar-EZ0 High Performance Sonar Range Finder", Available from: "http://www.maxbotix.com/documents/MB1000\_Datasheet.pdf", 2005.

[26] K. K. Shung and G. A. Thieme. "Ultrasonic Scattering in Biological Tissues". CRC, N.W. Boca Raton, Florida, 1992.

[27] L. Vergara, R. Miralles, J. Gosalbez, J. V. Fuente, U. L. Gomez, J. JAnaya, M. G. Hernandez and M. A. Izquierdo. "On estimating concrete porosity by ultrasonic signal processing techniques". In 17th ICA, Roma, September 2001.

[28] M. J. W. Povey. "Rapid Determination of Food Material Properties, Ultrasound in Food Processing". Blackie Academic I\& Professional, London, Wenheim, New York, 1997.

[29] L. Kleeman and R. Kuc, "Mobile robot sonar for target localisation and classification", The international Journal of Robotics Research, Vol. 14, No. 4,pp. 295--318, 1995.

[30] D. DeFatta, J. LUcas, and W. Hodgkiss, "Digital Signal Processing". Wiley, 1988.

[31] B. Barchan and R. Kuck, "Differentiating sonar reflections from corners and planes by employing an intelligent sensor", IEEE Transaction on Pattern Analysis and Machine Intelligence, Vol. 12, No. 6, pp. 560--569, 1990.

[32] I. E. Dror, M. Zagaeski and C. F. Moss, "Three-dimensional target recognition via sonar: a neural network model", Neural Networks, Vol. 8, No. 1, pp. 149--160, 1995.

[33] S. Bae, L. Udpa, and S. Udpa, "Classification of Ultrasonic Weld Inspection Data Using Principal Component Analysis", Review of Progress in Quantitative Nondestructive Evaluation, vol. 16, pp. 741-748, 1997.

[34] D. Berry, L. Udpa, and S. S. Udpa, "Classification of Ultrasonic Signals via Neural Networks", Review of Progress in Quantitative Nondestructive Evaluation, vol. 10A, pp. 659--666, 1991.

[35] L. M. Brown and R. DeNale, "Classification of Ultrasonic Defect Signatures Using An Artificial Neural Network", Review of Progress in Quantitative Nondestructive Evaluation, vol. 10A, pp. 705--712, 1991.

[36] H. Hamadene, and E. Colle, "A method based on neural networks for the recognition of the environment scanned by ultrasonic sensor", EUFIT'96 Fourth European Congress on Intelligent Techniques and soft Computing, Vol 1, Aachen, Germany, pp. 249--254, September 2-5, 1996.

[37] G. Lindstedt, and G. Olsson, "Using ultrasonics for sensing in a robotic Environment", IEEE, 1993.

[38] H. Peremans, J. M. K. Audenaert and V. Campenhout, "A high resolution sensor based on tri-aural perception", IEEE Transaction on Robotics ans Automation, Vol. 9, N. 1, pp. 36--48, 1993.

[39] K. R. Rao, "Discrete Transforms and their Applications". Van Nostrand Reinhold, New York, 1985.

[40] R. Kuc, "Introduction to Digital Signal Processing". McGraw-Hill, 1988.

[41] J. B. Santos and F. Perdigao, "Automatic defects classification - a contribution", NDT I\& E International, vol. 34, pp. 313--318, 2001.

[42] S. J. Song, H. J. Kim, and H. Cho, "Development of an intelligent system for ultrasonic flaw classification in weldments", Neuclear Engineering and Design, vol. 212, pp. 307--320, 2002.

[43] V. Vapnik, "Statistical Learning Theory, wiley Interscience publication", 1998.

[44] R. M. Haralick, K. Shanmugam, and I. Dinstein. "Textural features for image classification". IEEE Transactions on Systems, Man and Cybernetics, Vol. 3, No. 6, pp. 610-621, Nov 1973.

[45] URA-Sfax, Available from: "http://www.ura-sfax.org/fr/regional.php", 2006.

[46] G. H. John, "Enhancements to the Data Mining Process",thesis, 1997.

[47] A. C. Scherlen, J. C. Dumas, B. Guedj, A. Vignot, "'RecognizeCane": The new concept of a cane which recognizes the most common objects and safety clues", Proceedings of the 29th Annual International Conference of the IEEE EMBS, France, pp 63566359, 23-26 August 2007. 
